Math. Nachr. 185 (1997), 33-47

\title{
Perturbations of Banach Frames and Atomic Decompositions
}

By Ole Christensen of Lyngby and Christopher Heil of Atlanta

(Received September 21, 1994)

(Revised Version May 10, 1995)

\begin{abstract}
Banach frames and atomic decompositions are sequences that have basis-like properties but which need not be bases. In particular, they allow elements of a Banach space to be written as linear combinations of the frame or atomic decomposition elements in a stable manner. In this paper we prove several functional-analytic properties of these decompositions, and show how these properties apply to Gabor and wavelet systems. We first prove that frames and atomic decompositions are stable under small perturbations. This is inspired by corresponding classical perturbation results for bases, including the Paley-Wiener basis stability criteria and the perturbation theorem of Kato. We introduce new and weaker conditions which ensure the desired stability. We then prove duality properties of atomic decompositions and consider some consequences for Hilbert frames. Finally, we demonstrate how our results apply in the practical case of Gabor systems in weighted $L^{2}$ spaces. Such systems can form atomic decompositions for $L_{w}^{2}(\mathbb{R})$, but cannot form Hilbert frames for $L_{w}^{2}(\mathbb{R})$ unless the weight is trivial.
\end{abstract}

\section{Introduction}

Frames for Hilbert spaces were introduced by DUfFIN and SchaEfFER [DS] as part of their seminal research in nonharmonic Fourier series. Daubechies, Grossmann, and Meyer [DGM] later found a fundamental new application, to wavelet and Gabor transforms. Frames continue to play an important role in each of these areas.

A set of vectors $\left\{y_{i}\right\}$ in a Hilbert space $H$ is a (Hilbert) frame if the norms $\|x\|_{H}$ and $\left\|\left\{\left\langle x, y_{i}\right\rangle\right\}\right\|_{\ell^{2}}$ are equivalent. Define $U x=\left\{\left\langle x, y_{i}\right\rangle\right\}$. Then $U^{*} U x=\sum\left\langle x, y_{i}\right\rangle y_{i}$ is a continuous invertible mapping of $H$ onto itself. With $\tilde{y}_{i}=\left(U^{*} U\right)^{-1} y_{i}$, we have the reconstruction formulas

$$
x=\sum\left\langle x, \tilde{y}_{i}\right\rangle y_{i}=\sum\left\langle x, y_{i}\right\rangle \tilde{y}_{i}
$$

1991 Mathematics Subject Classification. Primary 42C99; Secondary 46B99, 46C99

Keywords and phrases. Atomic decompositions, Banach frames, frames, Gabor systems, perturbations. 
The collection $\left\{\tilde{y}_{i}\right\}$ also forms a frame, the dual frame of $\left\{y_{i}\right\}$. The representations in equation (1.1) need not be unique, i.e., $\left\{y_{i}\right\}$ need not be a basis. A frame which is a basis must be a Riesz basis. Conversely, all Riesz bases are frames. The basic theory of frames in Hilbert spaces can be found in DufFin and SchaEfFER's original paper [DS], YounG's classic text [Y], DAUBEChIES' paper [D1] and book [D2], or the research-tutorial [HW].

Frames were extended to Banach spaces by Gröchenig [G]. In Hilbert spaces, the norm equivalence hypothesis leads immediately to the reconstruction formula (1.1). This does not hold in Banach spaces in general. A decomposition of a Banach space is therefore defined as follows.

Definition 1.1. Let $X$ be a Banach space and let $X_{d}$ be an associated Banach space of scalar-valued sequences indexed by $\mathbb{N}=\{1,2,3, \ldots\}$. Let $\left\{y_{i}\right\}_{i \in \mathbb{N}} \subset X^{\prime}$ and $\left\{x_{i}\right\}_{i \in \mathbb{N}} \subset X$ be given. If:

(a) $\left\{\left\langle x, y_{i}\right\rangle\right\} \in X_{d}$ for each $x \in X$,

(b) the norms $\|x\|_{X}$ and $\left\|\left\{\left\langle x, y_{i}\right\rangle\right\}\right\|_{X_{d}}$ are equivalent, and

(c) $x=\sum_{i=1}^{\infty}\left\langle x, y_{i}\right\rangle x_{i}$ for each $x \in X$,

then $\left(\left\{y_{i}\right\},\left\{x_{i}\right\}\right)$ is a (linear) atomic decomposition of $X$ with respect to $X_{d}$. If the norm equivalence is given by $A\|x\|_{X} \leq\left\|\left\{\left\langle x, y_{i}\right\rangle\right\}\right\|_{X_{d}} \leq B\|x\|_{X}$, then $A, B$ are a choice of atomic bounds for $\left(\left\{y_{i}\right\},\left\{x_{i}\right\}\right)$.

Atomic decompositions have played a key role in the recent development of wavelet theory and Gabor, or windowed Fourier, analysis. Prior to the discovery of smooth wavelet orthonormal bases for the Hilbert space $L^{2}(\mathbb{R})$ in $[\mathrm{M}]$ and prior to the construction of wavelet and Gabor frames for $L^{2}(\mathbb{R})$ in $[D G M]$, FRAZIER and JAwERTH had constructed wavelet atomic decompositions for Besov spaces in [FJ], which they termed the " $\phi$-transform." FEICHTINGER constructed Gabor atomic decompositions for the modulation spaces in [F2]. These are Banach spaces similar in many respects to Besov spaces, defined by smoothness and decay conditions. WALnUT studied this construction in detail in $[\mathrm{W}]$, extending and improving many results for the case of weighted $L^{2}$ spaces. In particular, he showed that Gabor atomic decompositions in $L_{w}^{2}(\mathbb{R})$ need not be Hilbert frames. FEIChtinger and Gröchenig have developed a general theory which applies to an extremely broad class of function spaces and group representations [FG1], [FG2], [G].

In this paper we show that several new results on atomic decompositions follow simply once they are considered from a functional analytic point of view. We begin in Section 2 by demonstrating that atomic decompositions and the related concept of Banach frames are stable under small perturbations. This is inspired by corresponding classical perturbation results for bases, e.g., the PALEY-WIENER basis stability criteria $[\mathrm{PW}],[\mathrm{Y}]$ and the perturbation theorem of KATO [K]. The paper by RETHERFord and Holub $[\mathrm{RH}]$ is an excellent survey of perturbation results for bases. We introduce new and weaker conditions which ensure the desired stability. Next, we prove duality properties of atomic decompositions in Section 3, and consider some consequences for Hilbert frames in Section 4. Related Hilbert space results, partially inspired by a weak version of Theorem 2.3 first proved in [He], have appeared in [C1], [C2], [C3], and these have formed the basis for additional results by FAVIER and ZALIK [FZ]. We point out an implication for wavelet frames in $L^{2}(\mathbb{R})$ by connecting to a result of CHUI and SHI 
from [CS]. We also discuss the relationship between the hypotheses in our theorems to unconditional convergence of series, and prove a result on unconditional convergence which HoLuB has used in his recent characterization of near-Riesz bases in Hilbert spaces [Ho]. Finally, in Section 5 we demonstrate how our results can be applied to the practical case of Gabor atomic decompositions in weighted $L^{2}$ spaces. We show that no Gabor system can be a Hilbert frame for $L_{w}^{2}(\mathbb{R})$ unless the weight is trivial (bounded both above and below). Therefore atomic decompositions are a required tool for studying Gabor systems in weighted $L^{2}$ spaces.

The coherent state atomic decompositions are the most important for applications. These include both the wavelet and Gabor cases. A coherent state atomic decomposition arises from a group $G$ acting on a Banach space $X$ via a group representation $\pi$. We assume that $\pi(g)$ is a continuous invertible mapping of $X$ onto itself for each $g \in G$. In the wavelet case, the group is the affine group $\mathbf{A}=(\mathbb{R} \backslash\{0\}) \times \mathbb{R}$, and the group representation $\sigma$ is obtained by forming time-scale translates of functions $\psi$ :

$$
\sigma(a, b) \psi(t)=C(a, b) \psi(a t-b), \quad(a, b) \in \mathbf{A},
$$

with $C(a, b)$ chosen appropriately for $X$. For the Gabor case, the group is the Heisenberg group $\mathbf{H}=\mathbf{T} \times \mathbb{R} \times \mathbb{R}$, and the group representation $\rho$ is obtained via timefrequency translates of functions $f$ :

$$
\rho(z, a, b) f(t)=C(z, a, b) e^{2 \pi i b t} f(t-a), \quad(z, a, b) \in \mathbf{H} .
$$

Given such a function space $X$, group $G$, and representation $\pi$, we select a generator $x \in X$ (often called, by an abuse of terminology, the mother wavelet) and a discrete set $\left\{g_{i}\right\} \subset G$. If $\left\{\pi\left(g_{i}\right) x\right\}$ forms an atomic decomposition for $X$, then it is called a coherent state atomic decomposition. The discrete set $\left\{g_{i}\right\}$ is commonly chosen to be a "regular" subset of the group. For example, a wavelet coherent state decomposition usually has the form $\left\{a^{n / 2} \psi\left(a^{n} t-m b\right)\right\}_{m, n \in \mathbb{Z}}$ while a Gabor coherent state decomposition usually has the form $\left\{e^{2 \pi i m b t} f(t-n a)\right\}_{m, n \in \mathbb{Z}}$. The relationship of the affine and Heisenberg groups to wavelet and Gabor analysis on $L^{2}(\mathbb{R})$ is surveyed in detail in [HW].

We will use the following notations and terminology throughout. We assume that sequences are indexed by $\mathbb{N}=\{1,2,3, \ldots\}$. We say that a sequence $\left\{c_{i}\right\}$ is finite if only finitely many components are nonzero. A Banach space $X_{d}$ of sequences is solid if whenever $\left\{b_{i}\right\}$ and $\left\{c_{i}\right\}$ are sequences with $\left\{c_{i}\right\} \in X_{d}$ and $\left|b_{i}\right| \leq\left|c_{i}\right|$ then it follows that $\left\{b_{i}\right\} \in X_{d}$ and $\left\|\left\{b_{i}\right\}\right\|_{X_{d}} \leq\left\|\left\{c_{i}\right\}\right\|_{X_{d}}$. For example, let $e_{j}$ denote the delta sequence $e_{j}(i)=\delta_{i j}$. If $\left\{e_{j}\right\}_{j \in \mathbb{N}}$ forms an unconditional basis for $X_{d}$ then $X_{d}$ is solid. It also follows in this case that $X_{d}$ has an absolutely continuous norm, i.e., if $\left\{c_{i}\right\} \in X_{d}$ then $\lim _{n \rightarrow \infty}\left\|\left\{c_{i}-c_{i} \cdot \chi_{I_{n}}(i)\right\}\right\|_{X_{d}}=0$, where $\left\{I_{n}\right\}$ is any family of subsets of $\mathbb{N}$ such that $I_{1} \subset I_{2} \subset \cdots \nearrow \mathbb{N}$ and $\chi_{I_{n}}$ is the characteristic function $\chi_{I_{n}}(i)=1$ if $i \in I_{n}, 0$ if $i \notin I_{n}$. In particular, the hypotheses on $X_{d}, X_{d}^{\prime}$ in most of our results are satisfied if $X_{d}^{\prime}$ can be realized as a Banach space of sequences of scalars and if $\left\{e_{j}\right\}$ forms an unconditional basis for both $X_{d}$ and $X_{d}^{\prime}$. 


\section{Perturbation results}

An atomic decomposition provides a factorization of the identity map $I$ on $X$. That is, $I$ is written as a composition of the coefficient mapping $x \mapsto\left\{\left\langle x, y_{i}\right\rangle\right\}$ and the reconstruction operator $\left\{c_{i}\right\} \mapsto \sum c_{i} x_{i}$. Such series reconstructions are theoretically appealing. However, for numerical implementations it is often preferable to formulate the reconstruction operator via an iteration or other algorithm. We therefore make the following definition, which allows freedom in the form of the reconstruction operator.

Definition 2.1. Let $X$ be a Banach space and let $X_{d}$ be an associated Banach space of scalar-valued sequences indexed by $\mathbb{N}$. Let $\left\{y_{i}\right\}_{i \in \mathbb{N}} \subset X^{\prime}$ and $S: X_{d} \rightarrow X$ be given. If:

(a) $\left\{\left\langle x, y_{i}\right\rangle\right\} \in X_{d}$ for each $x \in X$,

(b) The norms $\|x\|_{X}$ and $\left\|\left\{\left\langle x, y_{i}\right\rangle\right\}\right\|_{X_{d}}$ are equivalent, and

(c) $S$ is bounded and linear, and $S\left\{\left\langle x, y_{i}\right\rangle\right\}=x$ for each $x \in X$,

then $\left(\left\{y_{i}\right\}, S\right)$ is a Banach frame for $X$ with respect to $X_{d}$. The mapping $S$ is the reconstruction operator. If the norm equivalence is given by

$$
A\|x\|_{X} \leq\left\|\left\{\left\langle x, y_{i}\right\rangle\right\}\right\|_{X_{d}} \leq B\|x\|_{X},
$$

then $A, B$ are a choice of frame bounds for $\left(\left\{y_{i}\right\}, S\right)$.

Note that if $U: X \rightarrow X_{d}$ is the coefficient mapping defined by $U x=\left\{\left\langle x, y_{i}\right\rangle\right\}$ then $\|S\|^{-1},\|U\|$ are a choice of frame bounds for the Banach frame $\left(\left\{y_{i}\right\}, S\right)$.

We now show that Banach frames are stable under small perturbations of the frame elements.

Theorem 2.2. Let $\left(\left\{y_{i}\right\}, S\right)$ be a Banach frame for $X$ with respect to $X_{d}$. Let $\left\{z_{i}\right\} \subset X^{\prime}$. If there exist $\lambda, \mu \geq 0$ such that

(a) $\lambda\|U\|+\mu \leq\|S\|^{-1}$, and

(b) $\left\|\left\{\left\langle x, y_{i}-z_{i}\right\rangle\right\}\right\|_{X_{d}} \leq \lambda\left\|\left\{\left\langle x, y_{i}\right\rangle\right\}\right\|_{X_{d}}+\mu\|x\|_{X}$ for all $x \in X$,

then there exists a reconstruction operator $T$ such that $\left(\left\{z_{i}\right\}, T\right)$ is a Banach frame for $X$ with respect to $X_{d}$ with frame bounds $\|S\|^{-1}-(\lambda\|U\|+\mu),\|U\|+(\lambda\|U\|+\mu)$, where $U$ is the coefficient mapping $U x=\left\{\left\langle x, y_{i}\right\rangle\right\}$.

Proof. The hypotheses imply that the operator $V: X \rightarrow X_{d}$ defined by $V x=$ $\left\{\left\langle x, z_{i}\right\rangle\right\}$ is bounded and satisfies $\|U x-V x\|_{X_{d}} \leq \lambda\|U x\|_{X_{d}}+\mu\|x\|_{X}$ for all $x \in X$. Therefore,

$$
\|V x\|_{X_{d}} \leq(\|U\|+\lambda\|U\|+\mu)\|x\|_{X} .
$$

This establishes the upper frame bound. For the lower bound, observe that $S U=I$, SO

$$
\|I-S V\| \leq\|S\|\|U-V\| \leq\|S\|(\lambda\|U\|+\mu)<1
$$

Therefore $S V$ is invertible, and $\left\|(S V)^{-1}\right\| \leq(1-(\lambda\|U\|+\mu)\|S\|)^{-1}$. Finally, if we set $T=(S V)^{-1} S$ then $T V=I$, and

$$
\|x\|_{X} \leq\|T\|\|V x\|_{X_{d}} \leq \frac{\|S\|}{1-(\lambda\|U\|+\mu)\|S\|}\|V x\|_{X_{d}} .
$$


This gives the desired lower bound: $\left(\|S\|^{-1}-(\lambda\|U\|+\mu)\right)\|x\|_{X} \leq\|V x\|_{X_{d}}$.

The hypotheses in Theorem 2.2 are natural from the point of view of perturbation of operators: they mean that the operator $U-V$ is relatively bounded with respect to $U$ (see p. 181 of $[\mathrm{K}]$ ).

For atomic decompositions, we can perturb in $X$ instead of $X^{\prime}$. Our result is a "PALEY-Wiener Theorem for atomic decompositions" (see p. 38 of $[\mathrm{Y}]$ ).

Theorem 2.3. Suppose that $X_{d}$ has an absolutely continuous norm. Let $\left(\left\{y_{i}\right\},\left\{x_{i}\right\}\right)$ be an atomic decomposition of $X$ with respect to $X_{d}$ with bounds $A, B$. Let $\left\{w_{i}\right\} \subset X$. If there exist $\lambda, \mu \geq 0$ such that

(a) $\lambda+\mu B<1$, and

(b) $\left\|\sum c_{i}\left(x_{i}-w_{i}\right)\right\|_{X} \leq \lambda\left\|\sum c_{i} x_{i}\right\|_{X}+\mu\left\|\left\{c_{i}\right\}\right\|_{X_{d}}$ for any finite sequence $\left\{c_{i}\right\} \in X_{d}$, then there exists a family $\left\{z_{i}\right\} \subset X^{\prime}$ such that $\left(\left\{z_{i}\right\},\left\{w_{i}\right\}\right)$ is an atomic decomposition of $X$ with respect to $X_{d}$ with bounds $A(1+(\lambda+\mu B))^{-1}, B(1-(\lambda+\mu B))^{-1}$. Moreover, $\left\{w_{i}\right\}$ is a basis for $X$ if and only if $\left\{x_{i}\right\}$ is a basis for $X$.

Proof. Because of the assumption that $X_{d}$ has an absolutely continuous norm, the series $\sum\left\langle x, y_{i}\right\rangle w_{i}$ is convergent for any $x \in X$. If we define $T: X \rightarrow X$ by $T x=$ $\sum\left\langle x, y_{i}\right\rangle w_{i}$, then

$$
\|x-T x\|_{X} \leq \lambda\|x\|_{X}+\mu\left\|\left\{\left\langle x, y_{i}\right\rangle\right\}\right\|_{X_{d}} \leq(\lambda+\mu B)\|x\|_{X}
$$

for all $x \in X$. Therefore $\|I-T\|<1$, so $T$ is invertible. Define $z_{i}=\left(T^{-1}\right)^{*} y_{i}$. Then

$$
x=T T^{-1} x=\sum\left\langle T^{-1} x, y_{i}\right\rangle w_{i}=\sum\left\langle x, z_{i}\right\rangle w_{i} .
$$

Further,

$$
\frac{A}{\|T\|}\|x\|_{X} \leq A\left\|T^{-1} x\right\|_{X} \leq\left\|\left\{\left\langle T^{-1} x, y_{i}\right\rangle\right\}\right\|_{X_{d}} \leq B\left\|T^{-1} x\right\|_{X} \leq B\left\|T^{-1}\right\|\|x\|_{X},
$$

so $\left(\left\{z_{i}\right\},\left\{w_{i}\right\}\right)$ is an atomic decomposition of $X$ with respect to $X_{d}$. Since $\|T\| \leq$ $1+\lambda+\mu B$ and $\left\|T^{-1}\right\| \leq(1-(\lambda+\mu B))^{-1}$, the bounds are as claimed.

Finally, assume that $\left\{x_{i}\right\}$ is a basis for $X$. Then $\left\{x_{i}\right\}$ and $\left\{y_{i}\right\}$ are biorthonormal, so $T x_{j}=\sum\left\langle T^{-1} T x_{j}, y_{i}\right\rangle w_{i}=w_{j}$. Therefore $\left\{w_{i}\right\}$ is a basis since $T$ is invertible. Conversely, if $\left\{w_{i}\right\}$ is a basis then $T^{-1}$ maps it onto $\left\{x_{i}\right\}$.

In the terminology of KATO (p. 181 of $[\mathrm{K}]$ ), the hypotheses in Theorem 2.3 are that the operator $K: D(K) \subset X_{d} \rightarrow X$ defined by $K\left\{c_{i}\right\}=\sum c_{i}\left(x_{i}-w_{i}\right)$ is relatively bounded with respect to the operator $\left\{c_{i}\right\} \mapsto \sum c_{i} x_{i}$. It is natural to call $K$ the "perturbation operator," since, as we have seen, conditions on $K$ imply that " $\left\{w_{i}\right\}$ inherits decomposition properties from $\left\{x_{i}\right\}$." We apply Theorem 2.3 to Gabor atomic decompositions for weighted $L^{2}$-spaces in Section 5 .

Specific choices of $\lambda$ and $\mu$ in Theorem 2.3 give conditions in the style of classic results on basis perturbation. 
Corollary 2.4. Let $\left(\left\{y_{i}\right\},\left\{x_{i}\right\}\right)$ be an atomic decomposition of $X$ with respect to $X_{d}$ with bounds $A, B$. Assume that $X_{d}, X_{d}^{\prime}$ satisfy:

(a) $X_{d}$ has an absolutely continuous norm,

(b) $X_{d}^{\prime}$ is a solid Banach space of scalar-valued sequences, and

(c) the action of $\left\{c_{i}\right\} \in X_{d}^{\prime}$ on $\left\{b_{i}\right\} \in X_{d}$ is given by $\left\langle\left\{b_{i}\right\},\left\{c_{i}\right\}\right\rangle=\sum b_{i} \bar{c}_{i}$.

If $\left\{w_{i}\right\} \subset X$ is such that $R=\left\|\left\{\left\|x_{i}-w_{i}\right\|_{X}\right\}\right\|_{X_{d}^{\prime}}<B^{-1}$, then there exists a family $\left\{z_{i}\right\} \subset X^{\prime}$ such that $\left(\left\{z_{i}\right\},\left\{w_{i}\right\}\right)$ is an atomic decomposition of $X$ with respect to $X_{d}$ with bounds $A(1+R B)^{-1}, B(1-R B)^{-1}$.

Proof. The hypotheses imply that $\left\|\sum c_{i}\left(x_{i}-w_{i}\right)\right\|_{X} \leq R\left\|\left\{c_{i}\right\}\right\|_{X_{d}}$ for any finite sequence $\left\{c_{i}\right\} \in X_{d}$. Therefore we can apply Theorem 2.3 with $\lambda=0$ and $\mu=R$.

A major drawback of Corollary 2.4 is that it generally does not apply to the problem of perturbing the generator of a coherent state atomic decomposition $\left\{\pi\left(g_{i}\right) x\right\}$. For example, if $\pi(g)$ is an isometry for each $g$ (as in the standard wavelet or Gabor cases) and if the generator $x$ is perturbed to $w$, then $\left\|\pi\left(g_{i}\right) x-\pi\left(g_{i}\right) w\right\|_{X} \equiv\|x-w\|_{X}$ for all $i$. Hence $\left\|\left\{\left\|\pi\left(g_{i}\right) x-\pi\left(g_{i}\right) w\right\|_{X}\right\}\right\|_{X_{d}^{\prime}}$ will typically be infinite. On the other hand, the hypotheses of Theorem 2.3 may still be applicable. We discuss this further in Sections 4 and 5. Similar remarks apply to the problem of perturbing the discrete set $\left\{g_{i}\right\}$.

We say that a sequence $\left\{x_{i}\right\} \subset X$ is a Bessel sequence for $X^{\prime}$ with respect to $X_{d}^{\prime}$ if there exists a constant $D$ such that

$$
\left\|\left\{\left\langle x_{i}, y\right\rangle\right\}\right\|_{X_{d}^{\prime}} \leq D\|y\|_{X^{\prime}} \text { for all } y \in X^{\prime} .
$$

The constant $D$ is the Bessel bound. The following additional consequence of Theorem 2.3 is motivated by a useful result about Riesz bases in Hilbert spaces [Hi].

Corollary 2.5. Let $\left(\left\{y_{i}\right\},\left\{x_{i}\right\}\right)$ be an atomic decomposition of $X$ with respect to $X_{d}$ with bounds $A, B$, and such that $\left\{x_{i}\right\}$ is a Bessel sequence for $X^{\prime}$ with respect to $X_{d}^{\prime}$ with Bessel bound D. Assume that $X_{d}, X_{d}^{\prime}$ satisfy hypotheses (a), (b), and (c) of Corollary 2.4. Assume that there exists a family $\left\{T_{k}\right\}$ of bounded operators on $X$ and scalars $a_{i k}$ so that

$$
x_{i}-w_{i}=\sum_{k} a_{i k} T_{k} x_{i} \quad \text { for each } i .
$$

If

(a) $a_{k}=\sup _{i}\left|a_{i k}\right|<\infty$ for each $k$, and

(b) $\sum a_{k}\left\|T_{k}\right\|<(B D)^{-1}$,

then there exists a family $\left\{z_{i}\right\} \subset X^{\prime}$ such that $\left(\left\{z_{i}\right\},\left\{w_{i}\right\}\right)$ is an atomic decomposition of $X$ with respect to $X_{d}$ with bounds $A\left(1+B D \sum a_{k}\left\|T_{k}\right\|\right)^{-1}$ and $B\left(1-B D \sum a_{k}\left\|T_{k}\right\|\right)^{-1}$.

Proof. Given a finite sequence $\left\{c_{i}\right\} \in X_{d}$, we have

$$
\left\|\sum_{i} c_{i}\left(x_{i}-w_{i}\right)\right\|_{X}=\left\|\sum_{i} c_{i} \sum_{k} a_{i k} T_{k} x_{i}\right\|_{X} \leq \sum_{k}\left\|T_{k}\right\|\left\|\sum_{i} c_{i} a_{i k} x_{i}\right\|_{X} .
$$


Fix any $k$. Then

$$
\begin{aligned}
\left\|\sum_{i} c_{i} a_{i k} x_{i}\right\|_{X} & =\sup _{\|y\|_{X^{\prime}}=1}\left|\sum_{i} c_{i} a_{i k}\left\langle x_{i}, y\right\rangle\right| \\
& =\sup _{\|y\|_{X^{\prime}}=1}\left|\left\langle\left\{c_{i}\right\},\left\{\overline{a_{i k}\left\langle x_{i}, y\right\rangle}\right\}\right\rangle\right| \\
& \leq\left\|\left\{c_{i}\right\}\right\|_{X_{d}} \sup _{\|y\|_{X^{\prime}}=1}\left\|\left\{a_{i k}\left\langle x_{i}, y\right\rangle\right\}\right\|_{X_{d}^{\prime}} \\
& \leq D a_{k}\left\|\left\{c_{i}\right\}\right\|_{X_{d}},
\end{aligned}
$$

where we have used the fact that $X_{d}^{\prime}$ is solid. Hence

$$
\left\|\sum_{i} c_{i}\left(x_{i}-w_{i}\right)\right\|_{X} \leq D\left(\sum_{k} a_{k}\left\|T_{k}\right\|\right)\left\|\left\{c_{i}\right\}\right\|_{X_{d}}
$$

for every finite sequence $\left\{c_{i}\right\} \in X_{d}$. We can therefore apply Theorem 2.3 with $\lambda=0$ and $\mu=D \sum a_{k}\left\|T_{k}\right\|$.

\section{Duality for atomic decompositions}

If $\left\{x_{i}\right\}$ is a basis for $X$ with coefficient functionals $\left\{y_{i}\right\}$ then $\left\{y_{i}\right\}$ is a basis for $\overline{\operatorname{span}}\left\{y_{i}\right\} \subset X^{\prime}$ with coefficient functions $\left\{x_{i}\right\} \subset X^{\prime \prime}$. We investigate the analogous question for atomic decompositions in this section.

Theorem 3.1. Let $\left(\left\{y_{i}\right\},\left\{x_{i}\right\}\right)$ be an atomic decomposition of $X$ with respect to $X_{d}$. Assume $X_{d}, X_{d}^{\prime}$ satisfy:

(a) $X_{d}$ is solid,

(b) $X_{d}^{\prime}$ is a Banach space of scalar-valued sequences,

(c) the action of $\left\{c_{i}\right\} \in X_{d}^{\prime}$ on $\left\{b_{i}\right\} \in X_{d}$ is given by $\left\langle\left\{b_{i}\right\},\left\{c_{i}\right\}\right\rangle=\sum b_{i} \bar{c}_{i}$, and

(d) $X_{d}^{\prime}$ has an absolutely continuous norm.

If $\left\{x_{i}\right\}$ is a Bessel sequence for $X^{\prime}$ with respect to $X_{d}^{\prime}$ then $\left(\left\{x_{i}\right\},\left\{y_{i}\right\}\right)$ is an atomic decomposition of $X^{\prime}$ with respect to $X_{d}^{\prime}$.

Proof. The hypotheses given imply that $\sum c_{i} y_{i}$ converges in $X^{\prime}$ for every $\left\{c_{i}\right\} \in X_{d}^{\prime}$. In particular, since $\left\{x_{i}\right\}$ is a Bessel sequence, if $y \in X^{\prime}$ is fixed then $\left\{\left\langle x_{i}, y\right\rangle\right\} \in X_{d}^{\prime}$, so $\sum\left\langle x_{i}, y\right\rangle y_{i}$ converges in $X^{\prime}$. Moreover, if $x \in X$ then

$$
\left\langle x, \sum_{i}\left\langle x_{i}, y\right\rangle y_{i}\right\rangle=\left\langle\sum_{i}\left\langle x, y_{i}\right\rangle x_{i}, y\right\rangle=\langle x, y\rangle .
$$

Hence $\sum\left\langle x_{i}, y\right\rangle y_{i}=y$ for each $y \in X^{\prime}$. 
It remains only to show that there is a constant $C$ such that $C\|y\|_{X^{\prime}} \leq\left\|\left\{\left\langle x_{i}, y\right\rangle\right\}\right\|_{X_{d}^{\prime}}$ for all $y \in X^{\prime}$. However, if $y \in X^{\prime}$ then

$$
\begin{aligned}
\|y\|_{X^{\prime}} & =\sup _{\|x\|_{X}=1}|\langle x, y\rangle| \\
& =\sup _{\|x\|_{X}=1}\left|\sum\left\langle x, y_{i}\right\rangle\left\langle x_{i}, y\right\rangle\right| \\
& \leq \sup _{\|x\|_{X}=1}\left\|\left\{\left\langle x, y_{i}\right\rangle\right\}\right\|_{X_{d}}\left\|\left\{\left\langle x_{i}, y\right\rangle\right\}\right\|_{X_{d}^{\prime}} \\
& \leq B\left\|\left\{\left\langle x_{i}, y\right\rangle\right\}\right\|_{X_{d}^{\prime}} .
\end{aligned}
$$

The Bessel sequence hypothesis is clearly necessary. For example, suppose $\left(\left\{y_{i}\right\},\left\{x_{i}\right\}\right)$ is an atomic decomposition of $X$ with respect to $X_{d}$ and that $\left\{w_{j}\right\}$ is not a Bessel sequence for $X^{\prime}$ with respect to $X_{d}^{\prime}$. Define $z_{j}=0$ for each $j$; then

1. $\left(\left\{y_{i}\right\} \cup\left\{z_{j}\right\},\left\{x_{i}\right\} \cup\left\{w_{j}\right\}\right)$ is an atomic decomposition of $X$ with respect to $X_{d}$, although

2. $\left(\left\{x_{i}\right\} \cup\left\{w_{j}\right\},\left\{y_{i}\right\} \cup\left\{z_{j}\right\}\right)$ is not an atomic decomposition of $X^{\prime}$ with respect to $X_{d}^{\prime}$

\section{Frame decompositions in Hilbert spaces}

In this section we consider the case $X=H$, a separable Hilbert space, and $X_{d}=\ell^{2}$. For Hilbert frames, it is customary to use a definition of frame bounds slightly different from the one we gave for Banach frames in Definition 2.1. In particular, if $\left\{x_{i}\right\}$ is a Hilbert frame then the norm equivalence between $\|x\|_{H}$ and $\left\|\left\{\left\langle x, x_{i}\right\rangle\right\}\right\|_{\ell^{2}}$ is usually written

$$
A\|x\|_{H}^{2} \leq \sum_{i}\left|\left\langle x, x_{i}\right\rangle\right|^{2} \leq B\|x\|_{H}^{2} \quad \text { for all } x \in H,
$$

with these $A, B$ called the frame bounds. For clarity, we will refer to $A, B$ given by equation (4.1) as Hilbert frame bounds; they are the squares of the Banach frame bounds given in Definition 2.1. The following result is an immediate consequence of Theorem 2.2, but a direct proof is so simple that we include it.

Proposition 4.1. Let $\left\{x_{i}\right\}$ be a Hilbert frame with Hilbert frame bounds A, B. Let $\left\{w_{i}\right\} \subset H$. If there is an $R<A$ such that

$$
\sum_{i}\left|\left\langle x, x_{i}-w_{i}\right\rangle\right|^{2} \leq R\|x\|_{H}^{2} \quad \text { for all } x \in H,
$$

then $\left\{w_{i}\right\}$ is a Hilbert frame with Hilbert frame bounds $A(1-\sqrt{R / A})^{2}$ and $B(1+\sqrt{R / B})^{2}$. 
Proof. The triangle inequality in $\ell^{2}$ states that $\left\|\left\{\left\langle x, x_{i}\right\rangle\right\}\right\|_{\ell^{2}} \leq\left\|\left\{\left\langle x, x_{i}-\omega_{i}\right\rangle\right\}\right\|_{\ell^{2}}+$ $\left\|\left\{\left\langle x, \omega_{i}\right\rangle\right\}\right\|_{\ell^{2}}$. Therefore, $\sum_{i}\left|\left\langle x, \omega_{i}\right\rangle\right|^{2} \geq\left(A^{1 / 2}-R^{1 / 2}\right)^{2}\|x\|_{H}^{2}$, and a similar proof applies for the upper bound.

Despite its simplicity, Proposition 4.1 is quite useful for applications. In most cases it is more difficult to verify the lower frame condition than the upper one. Proposition 4.1 shows that the difficult problem reduces to the easier one in the case of perturbation: the family $\left\{w_{i}\right\}$ is a frame if the difference $\left\{x_{i}-w_{i}\right\}$ satisfies the upper condition with a sufficiently small bound. This is a weaker hypothesis than the standard basis-type assumption that $\sum\left\|x_{i}-w_{i}\right\|_{H}^{2}<A$. In particular, this latter hypothesis cannot be applied to the problem of perturbing the generator $x$ of a coherent state frame $\left\{\pi\left(g_{i}\right) x\right\}$ in typical cases, e.g., if if $\pi(g)$ is an isometry for all $g$. However, Proposition 4.1 does apply: it states that $\left\{\pi\left(g_{i}\right) w\right\}$ is a frame if the set of coherent states $\left\{\pi\left(g_{i}\right)(x-w)\right\}$ generated by $x-w$ is a Bessel sequence with bound less than $A$. FAVIER and ZALIK [FZ] apply Proposition 4.1 explicitly to the case of Gabor and wavelet frames for $L^{2}(\mathbb{R})$. There are other applications of Proposition 4.1 to problems in irregular sampling and wavelet theory in [FZ] and [C3].

Proposition 4.1 connects with additional known results about frames. For example, using Remark 1 of [CS] we obtain the following: if $\left\{a^{n / 2} f\left(a^{n} x-m b\right)\right\}_{m, n \in \mathbb{Z}}$ is a wavelet frame for $L^{2}(\mathbb{R})$ with bounds $A, B$, and if $g \in L^{2}(\mathbb{R})$ is a function such that $\operatorname{supp}(\hat{f}-\hat{g}) \subseteq[-\pi, \pi]$, and there exists a number $R<A$ such that

$$
\forall \gamma, \quad \frac{1}{b} \sum_{n}\left|\hat{f}\left(a^{n} \gamma\right)-\hat{g}\left(a^{n} \gamma\right)\right|^{2} \leq R,
$$

then $\left\{a^{n / 2} g\left(a^{n} x-m b\right)\right\}_{m, n \in \mathbb{Z}}$ is also a frame for $L^{2}(\mathbb{R})$.

We have already remarked on the importance of the perturbation operator $K$. For Hilbert frames we are able to prove another result where $K$ plays the main role.

Theorem 4.2. Let $\left\{x_{i}\right\}$ be a Hilbert frame for $H$, and let $\left\{w_{i}\right\} \subset H$. If $K\left\{c_{i}\right\}=$ $\sum c_{i}\left(w_{i}-x_{i}\right)$ is compact as an operator from $\ell^{2}$ into $H$, then $\left\{w_{i}\right\}$ is a Hilbert frame for $\overline{\operatorname{span}}\left\{w_{i}\right\}$.

Proof. Define $T: \ell^{2} \rightarrow H$ by $T\left\{c_{i}\right\}=\sum c_{i} x_{i}$. Since $\left\{x_{i}\right\}$ is a frame, we know that $T$ is bounded. In fact, $\|T\|^{2} \leq B$, the upper Hilbert frame bound for $\left\{x_{i}\right\}$. Hence $V=T+K$ is a bounded operator from $\ell^{2}$ into $H$. If $x \in H$ then we compute

$$
\sum\left|\left\langle x, w_{i}\right\rangle\right|^{2}=\left\|V^{*} x\right\|_{H}^{2} \leq\|T+K\|^{2}\|x\|_{H}^{2} \leq B\left(1+\frac{\|K\|}{\sqrt{B}}\right)\|x\|_{H}^{2} .
$$

This establishes that $\left\{w_{i}\right\}$ satisfies an upper frame bound. The hypothesis that $K$ is compact will give the existence of the lower frame bound, but not a concrete value for it.

By Theorem 2.1 of [C1], to show the existence of the lower frame bound for $\left\{w_{i}\right\}$ it suffices to show that the "frame operator" $V V^{*}$ for $\left\{w_{i}\right\}$ is surjective. Now,

$$
V V^{*}=S+T K^{*}+K T^{*}+K K^{*},
$$


where $S=T T^{*}$ is the frame operator for $\left\{x_{i}\right\}$. The operator

$$
\left(T K^{*}+K T^{*}+K K^{*}\right) S^{-1}
$$

is compact, so the operator $\left(T K^{*}+K T^{*}+K K^{*}\right) S^{-1}+I$ has closed range (see Theorem 4.23 of $[\mathrm{R}]$ ). Composing this with $S$, we see that $V V^{*}$ also has closed range.

Now consider $V V^{*}$ as an operator on the closed subspace $\overline{\operatorname{span}}\left\{w_{i}\right\}$. Here $V V^{*}$ is injective: if $x \in \overline{\operatorname{span}}\left\{w_{i}\right\}$ and $V V^{*} x=0$ then $\sum\left|\left\langle x, w_{i}\right\rangle\right|^{2}=\left\langle V V^{*} x, x\right\rangle=0$, whence $x=0$. Since $V V^{*}$ has a closed range we therefore have Range $\left(V V^{*}\right)=\left(N\left(V V^{*}\right)\right)^{\perp}=$ $\overline{\operatorname{span}}\left\{w_{i}\right\}$. Thus $V V^{*}$ is surjective, as desired, and hence $\left\{w_{i}\right\}$ is a frame for $\overline{\operatorname{span}}\left\{w_{i}\right\}$.

In particular, $\left\{w_{i}\right\}$ is a frame for $\overline{\operatorname{span}}\left\{w_{i}\right\}$ if $\sum\left\|x_{i}-w_{i}\right\|_{H}^{2}<\infty$. By Proposition 4.1, we know that if $\sum\left\|x_{i}-w_{i}\right\|_{H}^{2}<A$ (the lower Hilbert frame bound for $\left\{x_{i}\right\}$ ) then $\left\{w_{i}\right\}$ is a frame for $H$, and therefore $\overline{\operatorname{span}}\left\{w_{i}\right\}=H$. However, if we have merely the equality $\sum\left\|x_{i}-w_{i}\right\|_{H}^{2}=A$, it may happen that $\overline{\operatorname{span}}\left\{w_{i}\right\} \neq H$. For example, let $\left\{x_{i}\right\}$ be an orthonormal basis for $H$, and set $w_{1}=0, w_{i}=x_{i}$ for $i>1$.

Also, note that the condition (4.2) in Proposition 4.1 is precisely the statement that $\|K\|<\sqrt{A}$. If $\|K\| \geq \sqrt{A}$ then $\left\{w_{i}\right\}$ need not be a frame for $\overline{\operatorname{span}}\left\{w_{i}\right\}$. For example, if $\left\{x_{i}\right\}$ is an orthonormal basis for $H$ and we set $w_{i}=x_{i}+x_{i+1}$, then $\|K\|=A=1$ but $\left\{w_{i}\right\}$ is not a frame for $\overline{\operatorname{span}}\left\{w_{i}\right\}=H$.

Our next result establishes the relation between Hilbert frames and atomic decompositions in Hilbert spaces. Note that if $\left\{y_{i}\right\}$ is a Hilbert frame for $H$ then $\left(\left\{y_{i}\right\},\left\{\tilde{y}_{i}\right\}\right)$ is an atomic decomposition of $H$ with respect to $\ell^{2}$, where $\left\{\tilde{y}_{i}\right\}$ is the dual frame of $\left\{y_{i}\right\}$. The converse requires additional hypotheses.

Theorem 4.3. Let $\left(\left\{y_{i}\right\},\left\{x_{i}\right\}\right)$ be an atomic decomposition of $H$ with respect to $\ell^{2}$. Then the following statements hold.

(a) $\left\{y_{i}\right\}$ is a Hilbert frame for $H$.

(b) If $\left\{x_{i}\right\}$ is a Bessel sequence for $H$ with respect to $\ell^{2}$ then it is a Hilbert frame for $H$.

(c) Assume $\left\{x_{i}\right\}$ is a Bessel sequence for $H$ with respect to $\ell^{2}$. Define $U, V: H \rightarrow \ell^{2}$ by $U x=\left\{\left\langle x, x_{i}\right\rangle\right\}$ and $V x=\left\{\left\langle x, y_{i}\right\rangle\right\}$. Then $\left\{x_{i}\right\}$ is the dual frame of $\left\{y_{i}\right\}$ if and only if $\operatorname{Range}(U)=\operatorname{Range}(V)$.

Proof. Statement (a) follows immediately from the definition. For (b), the lower frame bound follows from Theorem 3.1, or directly from the computation

$$
\|x\|_{H}^{4}=\left(\sum_{i}\left\langle x, y_{i}\right\rangle\left\langle x_{i}, x\right\rangle\right)^{2} \leq \sum_{i}\left|\left\langle x, y_{i}\right\rangle\right|^{2} \sum_{i}\left|\left\langle x_{i}, x\right\rangle\right|^{2} \leq B^{2}\|x\|_{H}^{2} \sum_{i}\left|\left\langle x_{i}, x\right\rangle\right|^{2} .
$$

Finally, for (c), note that the reconstruction formula (1.1) implies $U^{*} V=V^{*} U=I$. Let $E=\operatorname{Range}(U)$. Since $U$ is injective and $U V^{*} U=U$, we have $\left.\left(U V^{*}\right)\right|_{E}=\left.I\right|_{E}$. If $E=$ Range $(V)$, this implies $U V^{*} V=V$. Therefore, given $x \in H$,

$$
\left\{\left\langle x, y_{i}\right\rangle\right\}=V x=U V^{*} V x=\left\{\left\langle V^{*} V x, x_{i}\right\rangle\right\}=\left\{\left\langle x, V^{*} V x_{i}\right\rangle\right\}
$$


In particular, we must have $y_{i}=V^{*} V x_{i}$, whence $x_{i}=\left(V^{*} V\right)^{-1} y_{i}$ and $\left\{x_{i}\right\}$ is the dual frame of $\left\{y_{i}\right\}$. Conversely, if $\left\{x_{i}\right\}$ is the dual frame of $\left\{y_{i}\right\}$ then $V x=U V^{*} V x$, so Range $(V)=$ Range $(U)$ since $V^{*} V$ is invertible.

It need not be the case that $\left\{x_{i}\right\}$ is the dual frame of $\left\{y_{i}\right\}$ even if $\left(\left\{y_{i}\right\},\left\{x_{i}\right\}\right)$ is an atomic decomposition of $H$ with respect to $\ell^{2}$ and $\left\{x_{i}\right\}$ is a Bessel sequence. For example, let $\left\{y_{i}\right\}$ and $\left\{z_{j}\right\}$ be two frames for $H$. Define $w_{j}=0$ for each $j$. Then $\left(\left\{y_{i}\right\} \cup\left\{z_{j}\right\},\left\{\tilde{y}_{i}\right\} \cup\left\{w_{j}\right\}\right)$ is an atomic decomposition of $H$ with respect to $\ell^{2}$, but the dual frame of $\left\{y_{i}\right\} \cup\left\{z_{j}\right\}$ is $\left\{\tilde{y}_{i}\right\} \cup\left\{\tilde{z}_{j}\right\}$.

We close this section with a note about convergence, which generalizes Proposition 6.2.19 of [He]. The hypotheses on $X_{d}, X_{d}^{\prime}$ used in most of our results were needed to ensure that series such as $\sum c_{i} y_{i}$ converge unconditionally for every $\left\{c_{i}\right\}$ in the appropriate sequence space. In the Hilbert setting, we know that if $\left\{y_{i}\right\}$ is a Hilbert frame then $\sum c_{i} y_{i}$ converges unconditionally in $H$ for every $\left\{c_{i}\right\} \in \ell^{2}$. In fact, this is true if $\left\{y_{i}\right\}$ is merely a Bessel sequence for $H$ with respect to $\ell^{2}$.

Moreover, if $\left\{y_{i}\right\}$ is an arbitrary sequence in $H$ and $\sum c_{i} y_{i}$ converges unconditionally, then Orlicz' Theorem (see p. 18 of [LT]) implies that $\sum\left|c_{i}\right|^{2}\left\|y_{i}\right\|_{H}^{2}=\sum\left\|c_{i} y_{i}\right\|_{H}^{2}<\infty$. Therefore, if $\left\{y_{i}\right\}$ is norm-bounded below (meaning inf $\left\|y_{i}\right\|_{H}>0$ ), then $\sum\left|c_{i}\right|^{2}<\infty$.

In particular, if $\left\{y_{i}\right\}$ is a Bessel sequence for $H$ with respect to $\ell^{2}$ and $\left\{y_{i}\right\}$ is normbounded below, then

$$
\left\{c_{i}\right\} \in \ell^{2} \Longleftrightarrow \sum_{i} c_{i} y_{i} \text { converges unconditionally in } H .
$$

Among other results, Holub [Ho] recently used our equation (4.3) to prove the following equivalence for a Hilbert frame $\left\{y_{i}\right\}$ which is norm-bounded below: $\left\{y_{i}\right\}$ is a near Riesz basis (a Riesz basis plus finitely many elements) if and only if

$$
\sum_{i} c_{i} y_{i} \text { converges in } H \Longleftrightarrow \sum_{i} c_{i} y_{i} \text { converges unconditionally in } H \text {. }
$$

It would be useful to obtain similar characterizations in the Banach space setting.

\section{Applications to weighted $L^{2}$-spaces}

A function $w: \mathbb{R} \rightarrow(0, \infty)$ is called a moderate weight if there exists a submultiplicative function $m$, i.e., $m(s+t) \leq m(s) m(t)$ for all $s, t$, such that $w(s+t) \leq m(s) w(t)$ for all $s, t$. Frequently one also assumes that $w(0)=1$, but this assumption will play no role in the sequel. Corresponding to a moderate weight $w$ is a weighted $L^{2}$-space

$$
L_{w}^{2}(\mathbb{R})=\left\{f:\|f\|_{L_{w}^{2}}=\left(\int_{-\infty}^{\infty}|f(t)|^{2} w(t) d t\right)^{1 / 2}<\infty\right\} .
$$

$L_{w}^{2}(\mathbb{R})$ is a Hilbert space with respect to the weighted inner product

$$
\langle f, g\rangle_{w}=\int f(t) \overline{g(t)} w(t) d t .
$$


It can also be considered as a Banach space with dual space $L_{1 / w}^{2}(\mathbb{R})$ when the duality is defined by $\langle f, g\rangle=\int f(t) \overline{g(t)} d t$ for $f \in L_{w}^{2}(\mathbb{R})$ and $g \in L_{1 / w}^{2}(\mathbb{R}) . \quad L_{w}^{2}(\mathbb{R})$ is translation invariant, i.e., $f \in L_{w}^{2}(\mathbb{R})$ implies $T_{a} f \in L_{w}^{2}(\mathbb{R})$ for any $a \in \mathbb{R}$, where $T_{a} f(t)=f(t-a)$. The same is true for the modulation operator $E_{b} f(t)=e^{2 \pi i b t} f(t)$. Both $E_{b}$ and $T_{a}$ are bounded operators on $L_{w}^{2}(\mathbb{R})$.

Given $g \in L_{w}^{2}(\mathbb{R})$ and fixed $a, b$, the Gabor system generated by $g$ is $\left\{g_{m b, n a}\right\}_{m, n \in \mathbb{Z} \text {, }}$ where $g_{p, q}(t)=E_{p} T_{q} g(t)=e^{2 \pi i p t} g(t-q)$. Gabor systems in $L^{2}(\mathbb{R})$ are discussed in detail in [BHW], [D1], [D2], [HW]. Gabor systems in weighted $L^{2}$ spaces and in the more general setting of the modulation spaces are discussed in [BW], [F2], [W].

Gabor systems are very useful in analyzing the local frequency content of a function. Feichtinger and WALnUt have shown that Gabor systems can be useful outside the setting of $L^{2}(\mathbb{R})$. However, as we now show, although $L_{w}^{2}(\mathbb{R})$ is a Hilbert space, no Gabor system can form a Hilbert frame for $L_{w}^{2}(\mathbb{R})$ unless $w$ is essentially bounded, in which case $L_{w}^{2}(\mathbb{R})=L^{2}(\mathbb{R})$.

Lemma 5.1. If the Gabor system $\left\{g_{m b, n a}\right\}$ is a Hilbert frame for $L_{w}^{2}(\mathbb{R})$ then $w$ is bounded above and below.

Proof. Let $A, B$ be the frame bounds for $\left\{g_{m b, n a}\right\}$. Let $I$ be any interval of length $1 / b$, and let $f \in L_{w}^{2}(\mathbb{R})$ be any function supported within $I$. Then the Plancherel formula for Fourier series implies

$$
\begin{aligned}
\sum_{m, n}\left|\left\langle f, g_{m b, n a}\right\rangle_{w}\right|^{2} & =\sum_{m, n}\left|\int_{I} f(t) \overline{g(t-n a)} w(t) e^{-2 \pi i m b t} d t\right| \\
& =\sum_{n} \int_{I}|f(t) g(t-n a) w(t)|^{2} d t .
\end{aligned}
$$

Set $G(t)=\sum|g(t-n a)|^{2}$. Considering equation (5.1) together with the frame bounds, we conclude that $A \leq G(t) w(t) \leq B$ a.e. on $I$, and therefore the same inequalities hold almost everywhere on $\mathbb{R}$. Since $G$ is nonnegative and $a$-periodic, we can find a subset $E \subset[-a / 2, a / 2]$ with positive measure such that $0<\alpha \leq G(t) \leq \beta<\infty$ for almost every $t \in E$. Also, since $w$ is moderate there must exist a constant $C>0$ such that

$$
\sup _{[n a-a / 2, n a+a / 2]} w(t) \leq C \inf _{[n a-a / 2, n a+a / 2]} w(t) \quad \text { for every } n,
$$

with $C$ independent of $n$ [W]. Since $G$ is $a$-periodic, we therefore have

$$
\sup _{[n a-a / 2, n a+a / 2]} w(t) \leq C \inf _{E+n a} w(t) \leq \frac{C}{\alpha} \inf _{E+n a} G(t) w(t) \leq \frac{B C}{\alpha} .
$$

Hence $w(t) \leq B C / \alpha$ a.e., and similarly $w(t) \geq A /(C \beta)$ a.e.

Although Hilbert frames for $L_{w}^{2}(\mathbb{R})$ are not possible for nontrivial weights, WALNUT $[\mathrm{BW}],[\mathrm{W}]$, building on ideas of Feichtinger [F2], has shown that it is possible to construct Gabor atomic decompositions for $L_{w}^{2}(\mathbb{R})$ in much the same spirit as the classical results on Gabor frames for $L^{2}(\mathbb{R})$. These atomic decompositions will not be 
Hilbert frames for $L_{w}^{2}(\mathbb{R})$. Yet as atomic decompositions they still provide all of the desirable features of frames, including the equivalence between the norm of a function $f \in L_{w}^{2}(\mathbb{R})$ and a sequence space norm of $\left\{\left\langle f, g_{m, n}\right\rangle\right\}$. The natural sequence space for Gabor atomic decompositions of $X=L_{w}^{2}(\mathbb{R})$ is the weighted sequence space $X_{d}=\ell_{v}^{2}$, where the discrete weight $v$ is defined by

$$
v(m, n)=\inf _{[n a-a / 2, n a+a / 2]} w(t)
$$

We can apply our perturbation results to Gabor atomic decompositions. Let

$$
Y=\left\{f: \int_{-\infty}^{\infty}|f(t)|\left\|T_{t}\right\| d t<\infty\right\} .
$$

This is a Banach space with respect to the natural norm. Spaces of this type have been defined and studied by Feichtinger and Gröchenig [F1], [FG1], [FG2] in great detail; they are Wiener amalgam spaces. In their notation, $Y=W\left(L^{1}, L_{\left\|T_{(\cdot)}\right\|}\right)$.

The following result is Corollary 2.3.8 of [W]:

Proposition 5.2. Let $g \in Y$ and let $b \leq 1$. Then there exists a constant $C$ such that

$$
\left\|\sum_{m, n} a_{m, n} g_{m b, n a}\right\|_{L_{w}^{2}} \leq C b^{-1 / 2}\|g\|_{Y}\left\|\left\{a_{m, n}\right\}\right\|_{\ell_{v}^{2}}
$$

for all sequences $\left\{a_{m, n}\right\} \in \ell_{v}^{2}$.

The value of $C$ is independent of $g$ and $b$ and can be estimated explicitly. Applying Theorem 2.3 with $\lambda=0$ and $\mu=C b^{-1 / 2}\|g-h\|_{Y}$, we obtain the following result, which improves a theorem of WALNUT by giving an explicit bound on $\|g-h\|_{Y}$.

Corollary 5.3. Assume that $\left\{g_{m b, n a}\right\}$ is an atomic decomposition for $L_{w}^{2}(\mathbb{R})$ with respect to $\ell_{v}^{2}$, with bounds $A, B$. If $g-h \in Y$ and $\|g-h\|_{Y}<b^{1 / 2}(B C)^{-1}$, then $\left\{h_{m b, n a}\right\}$ is also an atomic decomposition of $L_{w}^{2}(\mathbb{R})$.

\section{Acknowledgements}

We thank Fred Andrew, John Benedetto, Hans Feichtinger, Karlheinz Gröchenig, HENRIK STETKæR, and DAVID WALnUt for valuable discussions and insights. We also thank the anonymous referees for their useful remarks and comments.

The first - named author was supported by Austrian Science Foundation Project S7001 mat. The second-named author was partially supported by National Science Foundation Grant $D M S-9401340$.

\section{References}

[BHW] J. J. Benedetto, C. Heil, and D. Walnut: Differentiation and the Balian-Low Theorem, J. Fourier Anal. Appl. 1 (1995), 355-402. 
[BW] J. J. Benedetto and D. WAlnut: Gabor frames for $L^{2}$ and related spaces, in: Wavelets: Mathematics and Applications, J. J. Benedetto, M. W. Frazier (Eds.), 97-162, CRC Press, Boca Raton, FL, 1994.

[C1] O. Christensen: Frames and the Projection Method, Appl. Comp. Harm. Anal. 1 (1993), $50-53$.

[C2] O. Christensen: A Paley-Wiener Theorem for Frames, Proc. Amer. Math. Soc. 123 (1995), $2199-2202$

[C3] O. Christensen: Moment Froblems and Stability Results for Frames. Appl. Comp. Harm. Anal. 3 (1996), 82-86.

[CS] C. K. Chui and X. Shi: Bessel Sequences and Affine Frames, Appl. Comp. Harm. Anal. 1 (1993), 29-49.

[D1] I. Daubechies: The Wavelet Transform, Time-Frequency Localization and Signal Analysis, IEEE Trans. Inform. Theory 39 (1990), 961-1005.

[D2] I. Daubechies: Ten Lectures on Wavelets, SIAM Press, Philadelphia, 1992.

[DGM] I. Daubechies, A. Grossmann, and Y. Meyer: Painless Nonorthogonal Expansions, J. Math. Phys. 27 (1986), 1271-1283.

[DS] R. J. Duffin and A. C. Schaeffer: A Class of Nonharmonic Fourier Series, Trans. Amer. Math. Soc. 72 (1952), 341-366.

[FZ] S. J. FAvier and R. A. Zalik: On the Stability of Frames and Riesz Bases, Appl. Comp. Harm. Anal. 2 (1995), 160-173.

[F1] H. G. Feichtinger: Banach Convolution Algebras of Wiener Type, in: Functions, Series, Operators, Proc. Conf. Budapest, 35, Colloq. Math. Soc. János Bolyai, B. Sz.-Nagy, J. Szabados (Eds.), 509-524, North Holland, Amsterdam, 1983.

[F2] H. G. Feichtinger: Atomic Characterizations of Modulation Spaces through Gabor-Type Representations, Rocky Mountain J. Math. 19 (1989), 113-126.

[FG1] H. G. Feichtinger and K. Gröchenig: Banach Spaces Related to Integrable Group Representations and their Atomic Decompositions, I, J. Funct. Anal. 86 (1989), 307-340.

[FG2] H. G. Feichtinger and K. Gröchenig: Banach Spaces Related to Integrable Group Representations and their Atomic Decompositions, II, Monatshefte für Mathematik 108 (1989), $129-148$.

[FJ] M. Frazier and B. Jawerth: Decompositions of Besov Spaces, Indiana Univ. Math. J. 34 (1985), 777-799.

[G] K. Gröchenig: Describing functions: Atomic Decompositions versus Frames, Monatshefte für Mathematik 112 (1991), 1-41.

[He] C. HeIL: Wiener Amalgam Spaces in Generalized Harmonic Analysis and Wavelet Theory, Ph.D. Thesis, University of Maryland, College Park, MD, 1990.

[HW] C. Heil and D. Walnut: Continuous and Discrete Wavelet Transforms, SIAM Review 31 (1989), 628-666.

[Hi] J. R. Higgins: Completeness and Basis Properties of Sets of Special Functions, Cambridge University Press, 1976.

[Ho] J. R. Holub: Pre-Frame Operators, Besselian Frames, and Near-Riesz Bases in Hilbert Spaces, Proc. Amer. Math. Soc. 122 (1994), 779-785.

[K] T. Kato: Perturbation Theory for Linear Operators, Second Edition, Springer-Verlag, New York, 1976.

[LT] J. Lindenstrauss and L. Tzafriri: Classical Banach Spaces, I, Springer-Verlag, New York, 1977.

[M] Y. Meyer: Principe d'Incertitude, Bases Hilbertiennes et Algèbres d'Opérateurs, Séminaire Bourbaki 662 (1985-1986). 
[PW] R. E. A. C. Paley and N. Wiener: Fourier Transforms in the Complex Domain, American Mathematical Society Colloquium Publications Vol. 19, American Mathematical Society, New York, 1934.

[RH] J. R. Retherford and J. R. Holub: The Stability of Bases in Banach and Hilbert Spaces, J. Reine Angew. Math. 246 (1971), 136-146.

[R] W. Rudin: Functional Analysis, Second Edition, McGraw-Hill, New York, 1991.

[W] D. Walnut: Weyl-Heisenberg Wavelet Expansions: Existence and Stability in Weighted Spaces, Ph.D. Thesis, University of Maryland, College Park, MD, 1989.

[Y] R. Young: An Introduction to Nonharmonic Fourier Series, Academic Press, New York, 1980.

Department of Mathematics

Technical University of Denmark

Building 303

2800 Lyngby

Denmark

e-mail:

olechr@mat.dtu.dk
School of Mathematics

Georgia Institute of Technology

Atlanta, Georgia 30332-0160

USA 


\section{ERRATA}

Note: This errata listing is not included in the published version of this paper.

In the statement of Theorem 2.2 part (a), the inequality $\leq$ should be replaced by $<$. 